\title{
Umbrella review and multivariate meta-analysis of diagnostic test accuracy studies on hybrid non-invasive imaging for coronary artery disease
}

\author{
Giuseppe Biondi-Zoccai, MD, MStat, ${ }^{\text {a,b }}$ Francesco Versaci, MD, ${ }^{\mathrm{c}}$ Ami \\ E. Iskandrian, MD, MACC, ${ }^{d}$ Orazio Schillaci, $M D,{ }^{\text {b,e }}$ Alessandro \\ Nudi, MD, ${ }^{\mathrm{f}}$ Giacomo Frati, MD, MSc, ${ }^{\mathrm{a}, \mathrm{b}}$ and Francesco Nudi, MD ${ }^{\mathrm{f}, \mathrm{g}, \mathrm{h}}$ \\ a Department of Medico-Surgical Sciences and Biotechnologies, Sapienza University of Rome, \\ Latina, Italy \\ b IRCCS NEUROMED, Pozzilli, Italy \\ c Division of Cardiology, S. Maria Goretti Hospital, Latina, Italy \\ d Division of Cardiovascular Disease, University of Alabama at Birmingham, Birmingham, AL \\ e Department of Nuclear Medicine, Tor Vergata University, Rome, Italy \\ f Replycare, Rome, Italy \\ g Service of Nuclear Cardiology, Madonna della Fiducia Clinic, Rome, Italy \\ h Service of Nuclear Cardiology, Ostia Radiologica, Rome, Italy
}

Received Oct 3, 2018; accepted Oct 4, 2018

doi: $10.1007 / \mathrm{s} 12350-018-01487-\mathrm{w}$

Background. The diagnosis of coronary artery disease (CAD) remains challenging. It is uncertain whether hybrid imaging can improve diagnostic accuracy for CAD.

Methods. This is a systematic review and multivariate meta-analysis. We searched PubMed and The Cochrane Library for recent $(\geq 2010)$ systematic reviews of diagnostic test accuracy studies on non-invasive imaging for CAD. Study-level data were extracted from them, and pooled with pairwise and multivariate meta-analytic methods, using invasive coronary angiography (ICA) or invasive fractional flow reserve (FFR) as reference standards, focusing on sensitivity and specificity.

Results. Details from 661 original studies $(71,823$ patients) were pooled. Pairwise metaanalysis using ICA as reference showed that anatomic imaging was associated with the best diagnostic accuracy (sensitivity $=0.95$ [95\% confidence interval $0.94-0.96]$, specificity $=0.83$ [0.81-0.85]), whereas using FFR as reference identified hybrid imaging as the best test (sensitivity $=0.87[0.83-0.90]$, specificity $=0.82[0.76-0.87])$. Multivariate meta-analysis confirmed the superiority of anatomic imaging using ICA as reference (sensitivity $=0.96$, specificity $=0.83$ ), and hybrid imaging using FFR as reference (sensitivity $=0.88[0.86-0.91]$, specificity $=0.82[0.77-0.87])$.

Conclusions. Non-invasive hybrid imaging tests appear superior to anatomic or functional only tests to diagnose ischemia-provoking coronary lesions, whereas anatomic imaging is best to

Electronic supplementary material The online version of this article (https://doi.org/10.1007/s12350-018-01487-w) contains supplementary material, which is available to authorized users.

The authors of this article have provided a PowerPoint file, available for download at SpringerLink, which summarises the contents of the paper and is free for re-use at meetings and presentations. Search for the article DOI on SpringerLink.com.

Funding This work was supported by Replycare, Rome, Italy.
Reprint requests: Giuseppe Biondi-Zoccai, MD, MStat, Department of Medico-Surgical Sciences and Biotechnologies, Sapienza University

of Rome, Latina, Italy; giuseppe.biondizoccai@uniromal.it $1071-3581 / \$ 34.00$

Copyright (c) 2018 American Society of Nuclear Cardiology. 
diagnose and/or rule out angiographically significant CAD.

Systematic review registration. PROSPERO Registry Number CRD42018088528. (J Nucl Cardiol 2018)

Key Words: Coronary artery disease $\cdot$ Diagnosis $・$ Imaging $\cdot$ Multivariate meta-analysis $•$ Umbrella review

\begin{tabular}{|ll|}
\hline \multicolumn{2}{|l}{ Abbreviations } \\
+ LR & Positive likelihood ratio \\
- LR & Negative likelihood ratio \\
AUC & Area under the curve \\
CAD & Coronary artery disease \\
CI & Confidence interval \\
CMR & Cardiac magnetic resonance \\
CT & Computed tomography \\
CTFFR & Computed tomography- \\
& fractional flow reserve \\
CZT & Cadmium-zinc-telluride \\
DOR & Diagnostic odds ratio \\
ECG & Electrocardiography \\
FFR & Fractional flow reserve \\
FN & False negatives \\
FP & False positives \\
ICA & Invasive coronary angiography \\
MPI & Myocardial perfusion imaging \\
PET & Positron emission tomography \\
PROSPERO & International Prospective \\
& Register of Systematic Reviews \\
SPECT & Single photon emission \\
& computed tomography \\
SROC & Summary receiver-operating curve \\
TN & True negatives \\
TP & True positives \\
\hline & \\
\hline
\end{tabular}

\section{INTRODUCTION}

Coronary artery disease (CAD) is the most common and most important cause of ischemic heart disease, with exceedingly relevant implications on morbidity and mortality world-wide ${ }^{1-4}$ On top of key advances in understanding pathophysiology and newer prevention and management strategies, the last decades have witnessed momentous developments in methods for CAD diagnosis. These approaches could broadly be divided into those that examine coronary anatomy, physiology, or both. ${ }^{5-13}$ From exercise electrocardiography (ECG) testing, which has several limitations in terms of diagnostic and prognostic accuracy, ${ }^{6}$ there are now several appealing alternatives for non-invasive imaging tests for $\mathrm{CAD}$, distinguished in anatomical tests capable of describing the morphologic features of coronary vessels, i.e., computed tomography angiography (CTA), and functional tests capable of highlighting ischemia-provoking lesions, such as stress echocardiography, ${ }^{7}$ myocardial perfusion imaging (MPI) with single photon emission computed tomography (SPECT) ${ }^{8}$ MPI with positron emission tomography (PET), ${ }^{9}$ cardiac magnetic resonance (CMR),${ }^{11}$ computed tomography myocardial perfusion (CTP), ${ }^{12}$ and noninvasive assessment of the functional significance of a coronary stenosis with computed tomography-fractional flow reserve (CTFFR). ${ }^{13}$

Developments of improved image processing software enabled the integration of anatomic and functional tests of hybrid non-invasive imaging. Several studies in favor of this integrative strategy have already been reported and summarized elsewhere, ${ }^{14}$ but a comprehensive comparative analysis of their incremental and comparative diagnostic accuracy is lacking. We thus aimed to conduct an umbrella review and multivariate meta-analysis on diagnostic test accuracy studies of noninvasive imaging tests for $\mathrm{CAD}^{15-17}$ defined as anatomic, functional, or hybrid tests.

\section{METHODS}

\section{Design}

This umbrella review and network meta-analysis was designed in keeping with established standards, ${ }^{15-17}$ the Cochrane Collaboration recommendations, ${ }^{18}$ registered prospectively in the International Prospective Register of Systematic Reviews (PROSPERO, ID\#CRD42018088528), ${ }^{19}$ and reported according to the Preferred Reporting Items for Systematic Reviews and Meta-Analyses (PRISMA) guidelines. $^{20}$ All reviewing activities were conducted by two reviewers independently, with divergences solved after consensus.

\section{Search}

PubMed was searched for systematic reviews on diagnostic test accuracy on studies for CAD published since January 1, 2010 with the following string: (((ischaemic OR ischemic) AND heart) OR (coronary AND artery) AND disease) AND (sensitivity OR specificity OR (predictive AND value) OR (likelihood AND ratio)) AND ("systematic review" OR "systematic overview" OR meta-analysis[pt]) AND (“2010/01/01',[pdat]: “3000/12/31'”[pdat]). The Cochrane Library was also searched for suitable reviews. 
Searches were last updated on August 22, 2018. No language restriction was enforced.

\section{Selection, Abstraction, and Appraisal}

Citations were first screened at the title or abstract level. If deemed potentially pertinent (i.e., reporting on systematic reviews of diagnostic test accuracy studies for the non-invasive imaging diagnosis of $\mathrm{CAD}$ ), they were then extracted in full text. For each diagnostic comparison, the three most comprehensive and recent meta-analyses were selected for abstraction of study-level data. Older or less comprehensive reviews were instead discarded unless reporting on additional studies, as were duplicate reports or meta-analyses focusing on prevention, treatment, or prognosis.

From each shortlisted meta-analysis, we obtained key study-level details on design, sample, patients, reference standard used, false positives (FP), false negatives (FN), true positives (TP), and true negatives (TN), limiting ourselves to per-patient details (thus disregarding per-vessel and per-lesion details), and avoiding duplicate data entry. In addition, we did not include studies focusing only on non-SPECT scintigraphy or calcium scoring. Study-level validity was not appraised directly but we relied instead on the original methods and results used in each included meta-analysis.

The main pairwise and multivariate analyses were based on the anatomic vs. functional vs. hybrid comparison for index test, using invasive coronary angiography (ICA) and fractional flow reserve (FFR) as reference tests for, respectively, anatomical, and functional assessment of CAD. Ancillary analyses were conducted distinguishing as main alternative non-invasive imaging tests the following: CMR, CTA, CTP, PET, SPECT, and stress echocardiography, alone or combination as hybrid tests. Notably, the following combinations of anatomical-functional hybrid imaging were appraised: CMR/ CTA, CTP/CTA, computed tomography-fractional flow reserve (CTFFR), PET/CTA, SPECT/CTA, and stress echocardiography/CTA.

\section{Analysis}

Continuous variables are reported as median (minimum; maximum), and categorical variables as count (\%). We first computed sensitivity, specificity, positive likelihood ratio (+ LR), negative likelihood ratio ( $-\mathrm{LR})$, diagnostic odds ratio (DOR), and area under the curve (AUC) of summary receiver-operating curve (SROC) in a frequentist pairwise framework using a random effect model, eventually providing point estimates of pooled effect (95\% confidence intervals) (Online supplement). ${ }^{16}$

We then proceeded to multivariate meta-analysis of sensitivity, specificity, $+\mathrm{LR}$, and $-\mathrm{LR}$ in a frequentist framework with a random effects model, yielding point estimates of effects (95\% confidence intervals). ${ }^{17}$ Pairwise and multivariate statistical inconsistency was appraised with Cochran $Q$ and $\chi^{2}$ tests, with corresponding $P$ values for heterogeneity $\left(P_{\text {het }}\right)$ and Higgins $I^{2}$. Small study effects were appraised with asymmetry plots and tests according to Deeks et al. ${ }^{16}$ Statistical significance was set at the 2-tailed 0.10 level for $P_{\text {effect }}$ and $P_{\text {Deeks }}$. Computations were performed with R (R Foundation for Statistical Computing, Vienna, Austria) and Stata 13 (StataCorp, College Station, TX, USA).

\section{RESULTS}

\section{Included Reviews and Studies}

From an initial set of 701 potentially relevant citations, we finally included 22 pertinent meta-analyses (Figure 1S). ${ }^{14,21-41}$ Specifically, 19 were systematic reviews published in peer-reviewed journals, whereas 3 were health technology assessments produced in the UK or Canada (Table 1). The focus of these reviews was on the comparative diagnostic accuracy of diagnostic tests for CAD, using either ICA or FFR as reference tests. Reviews varied in comprehensiveness, as they included from 7 to 166 studies, and from 389 to 17,901 patients (Table 2). In view of their different scope, findings of reviews were different, with some supporting functional tests, others favoring anatomic tests, and others still concluding for the superiority of hybrid imaging (Table 2).

From these systematic reviews, we then extracted details on 661 original studies (71,823 patients) (Table 3). Included studies were published between 1982 and 2018, with a median of 2005, and ranged in sample size from 5 to 1853 , with a median of 73 . Most studies focused on the comparison of functional tests vs. ICA, or on the comparison between anatomic or functional tests vs. FFR (Figure 1).

\section{Pairwise Meta-analysis}

Pairwise meta-analyses using ICA as reference standard were characterized by significant statistical heterogeneity and inconsistency for all available comparisons (Table 1S) (Figures $2 \mathrm{~S}$ to $7 \mathrm{~S}$ ). Moreover, small study effects were evident for the analysis focusing on functional imaging vs ICA. Notwithstanding these limitations, we computed effect estimates, finding that anatomic imaging yielded the highest sensitivity $(0.95$ [95\% confidence interval 0.94-0.96]), with specificity $=0.83 \quad(0.81-0.85), \quad+\mathrm{LR}=5.7 \quad(5.0-6.5), \quad-$ $\mathrm{LR}=0.06$ (0.05-0.07), DOR $=94.7$ (73.1-122.8), and AUC $=0.99$ (0.99-0.99), whereas hybrid imaging was associated with the best specificity (0.89 [0.83-0.92], with sensitivity $=0.90(0.85-0.93), \quad+\mathrm{LR}=7.8$ (5.3$11.9), \quad-\mathrm{LR}=0.11 \quad(0.08-0.17), \quad$ DOR $=68.8 \quad$ (37.9124.8), and AUC = 0.98 (0.97-0.99) (Table 4).

Pairwise meta-analyses using FFR as reference standard were also fraught with significant heterogeneity and inconsistency, despite the non-significant results of 


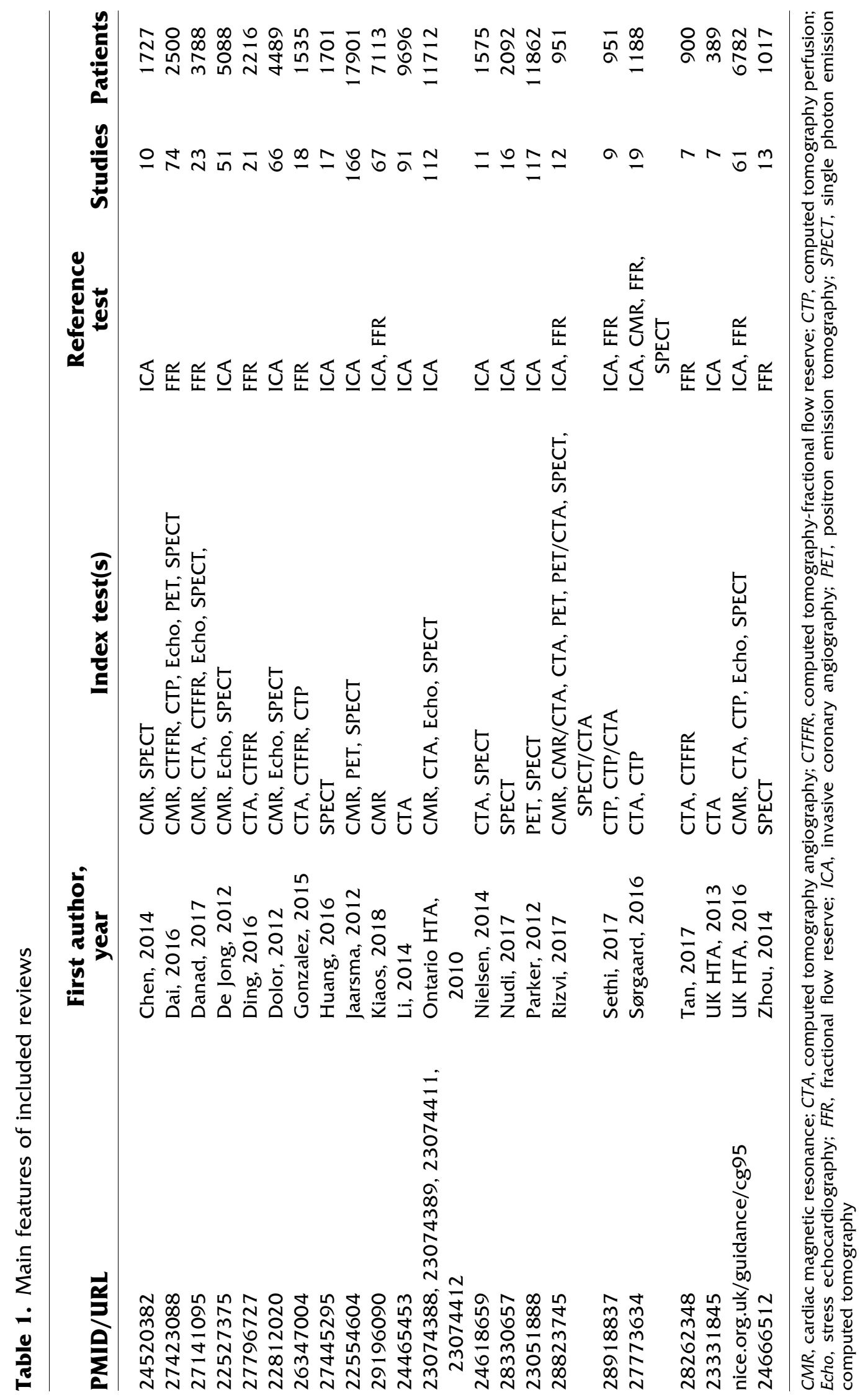


Table 2. Main findings of included systematic reviews

\begin{tabular}{|c|c|c|c|}
\hline $\begin{array}{l}\text { First } \\
\text { author, } \\
\text { year }\end{array}$ & Quality & Validity & Main findings \\
\hline Chen, 2014 & High & High & CMR is more sensitive than SPECT to detect anatomically significant CAD \\
\hline Dai, 2016 & Moderate & High & $\begin{array}{l}\text { CMR, CTFFR, CTP and PET have similar sensitivity and specificity to detect } \\
\text { functionally significant CAD }\end{array}$ \\
\hline Danad, 2017 & High & High & $\begin{array}{l}\text { CMR has the highest sensitivity and specificity to detect functionally } \\
\text { significant CAD }\end{array}$ \\
\hline $\begin{array}{l}\text { De Jong, } \\
2012\end{array}$ & High & High & $\begin{array}{l}\text { CMR has higher diagnostic accuracy than stress echocardiography and } \\
\text { SPECT }\end{array}$ \\
\hline Ding, 2016 & Mode & High & CTFFR has good diagnostic accuracy to detect functionally significant $C A D$ \\
\hline Dolor, 2012 & High & High & $\begin{array}{l}\text { CMR, CTA, stress echocardiography and SPECT have similar diagnostic } \\
\text { accuracy to detect anatomically significant CAD }\end{array}$ \\
\hline $\begin{array}{l}\text { Gonzalez, } \\
2015\end{array}$ & Moderate & High & $\begin{array}{l}\text { Both CTFFR and CTP improve the diagnostic accuracy of CTA to detect } \\
\text { functionally significant CAD }\end{array}$ \\
\hline Huang, 2016 & Moderate & Moderate & $\begin{array}{l}\text { Attenuation correction improves the sensitivity of SPECT to detect } \\
\text { anatomically significant CAD }\end{array}$ \\
\hline $\begin{array}{l}\text { Jaarsma, } \\
2012\end{array}$ & High & High & $\begin{array}{l}\text { PET, CMR, and SPECT have decreasing diagnostic accuracy to detect } \\
\text { anatomically significant CAD }\end{array}$ \\
\hline Kiaos, 2018 & High & High & $\begin{array}{l}\text { CMR has higher diagnostic accuracy to detect functionally significant } \\
\text { rather than anatomically significant CAD }\end{array}$ \\
\hline $\mathrm{Li}, 2014$ & High & High & $\begin{array}{l}\text { The diagnostic accuracy of CTA to detect anatomically significant CAD } \\
\text { does not improve with more than } 64 \text { detectors }\end{array}$ \\
\hline $\begin{array}{l}\text { Ontario HTA, } \\
2010\end{array}$ & High & High & $\begin{array}{l}\text { CMR and CTA have higher diagnostic accuracy than stress } \\
\text { echocardiography and SPECT to detect anatomically significant CAD }\end{array}$ \\
\hline Nielsen, 2014 & Moderate & High & $\begin{array}{l}\text { CTA has higher diagnostic accuracy than SPECT to detect anatomically } \\
\text { significant CAD }\end{array}$ \\
\hline Nudi, 2017 & High & High & $\begin{array}{l}\text { SPECT with cadmiu-zinc-telluride cameras has good sensitivity but } \\
\text { suboptimal specificity to detect anatomically significant CAD }\end{array}$ \\
\hline Parker, 2012 & High & High & $\begin{array}{l}\text { PET has higher sensitivity but similar specificity to SPECT to detect } \\
\text { anatomically significant CAD }\end{array}$ \\
\hline Rizvi, 2017 & Moderate & High & $\begin{array}{l}\text { Hybrid anatomo-functional imaging improves the specificity of CTA to } \\
\text { detect anatomically or functionally significant CAD }\end{array}$ \\
\hline Sethi, 2017 & Moderate & Moderate & CTP improves the specificity of CTA to detect functionally significant CAD \\
\hline $\begin{array}{l}\text { Sørgaard, } \\
2016\end{array}$ & Moderate & High & $\begin{array}{l}\text { CTP has good diagnostic accuracy to detect functionally significant CAD, } \\
\text { which further improves with CTP/CTA }\end{array}$ \\
\hline Tan, 2017 & Moderate & Moderate & $\begin{array}{l}\text { CTFFR improves the specificity of CTA to detect functionally significant } \\
\text { CAD }\end{array}$ \\
\hline $\begin{array}{l}\text { UK HTA, } \\
2013\end{array}$ & High & High & CTA has good diagnostic accuracy to detect anatomically significant CAD \\
\hline $\begin{array}{l}\text { UK HTA, } \\
2016\end{array}$ & High & High & $\begin{array}{l}\text { CTA has higher sensitivity than other non-invasive diagnostic modalities } \\
\text { to detect anatomically significant CAD }\end{array}$ \\
\hline Zhou, 2014 & High & High & $\begin{array}{l}\text { SPECT has moderate diagnostic accuracy to detect functionally significant } \\
\text { CAD }\end{array}$ \\
\hline
\end{tabular}

$C A D$, coronary artery disease; CMR, cardiac magnetic resonance; CTA, computed tomography angiography; CTFFR, computed tomography-fractional flow reserve; CTP, computed tomography perfusion; Echo, stress echocardiography; PET, positron emission tomography; SPECT, single photon emission computed tomography 


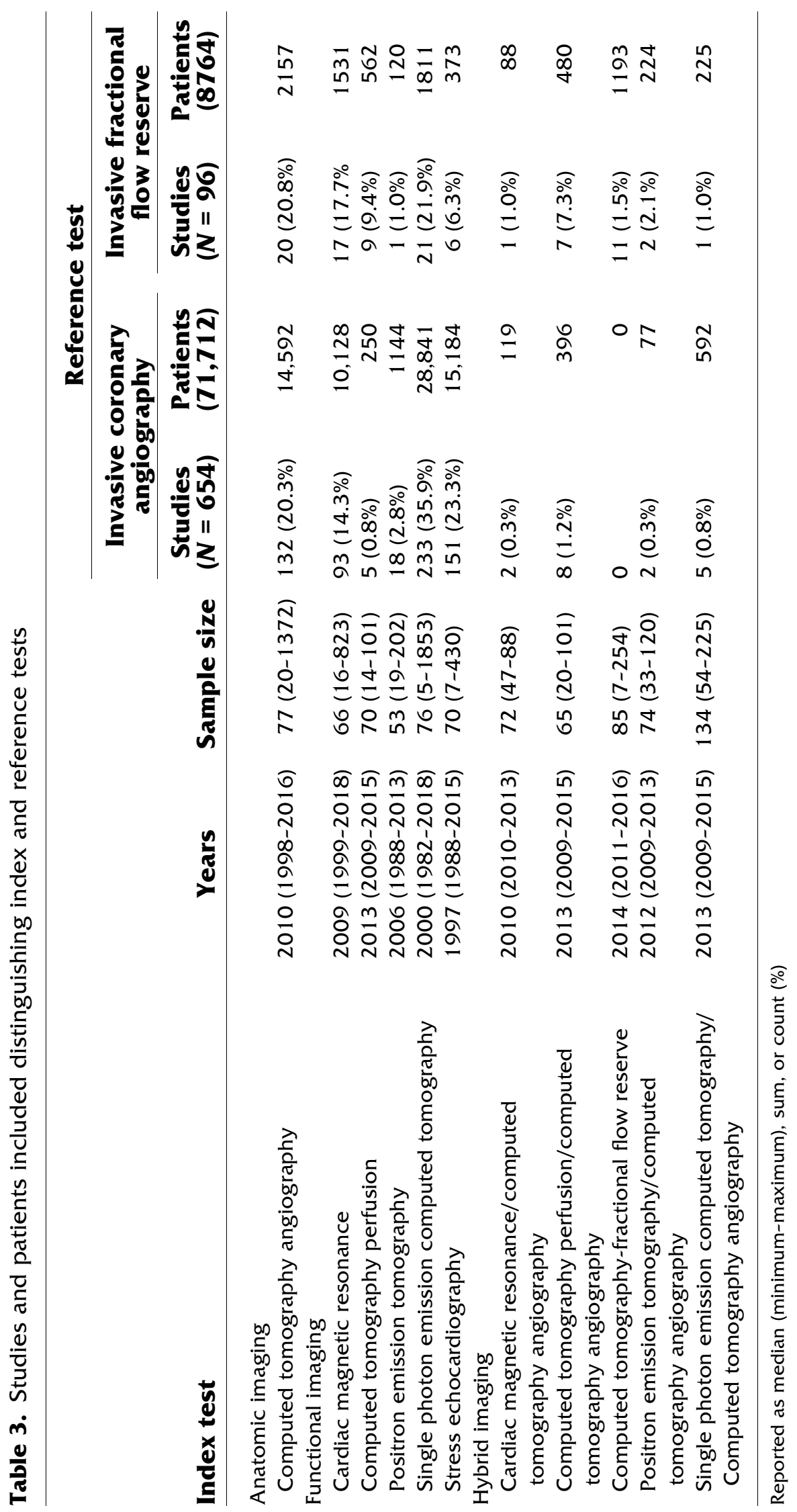




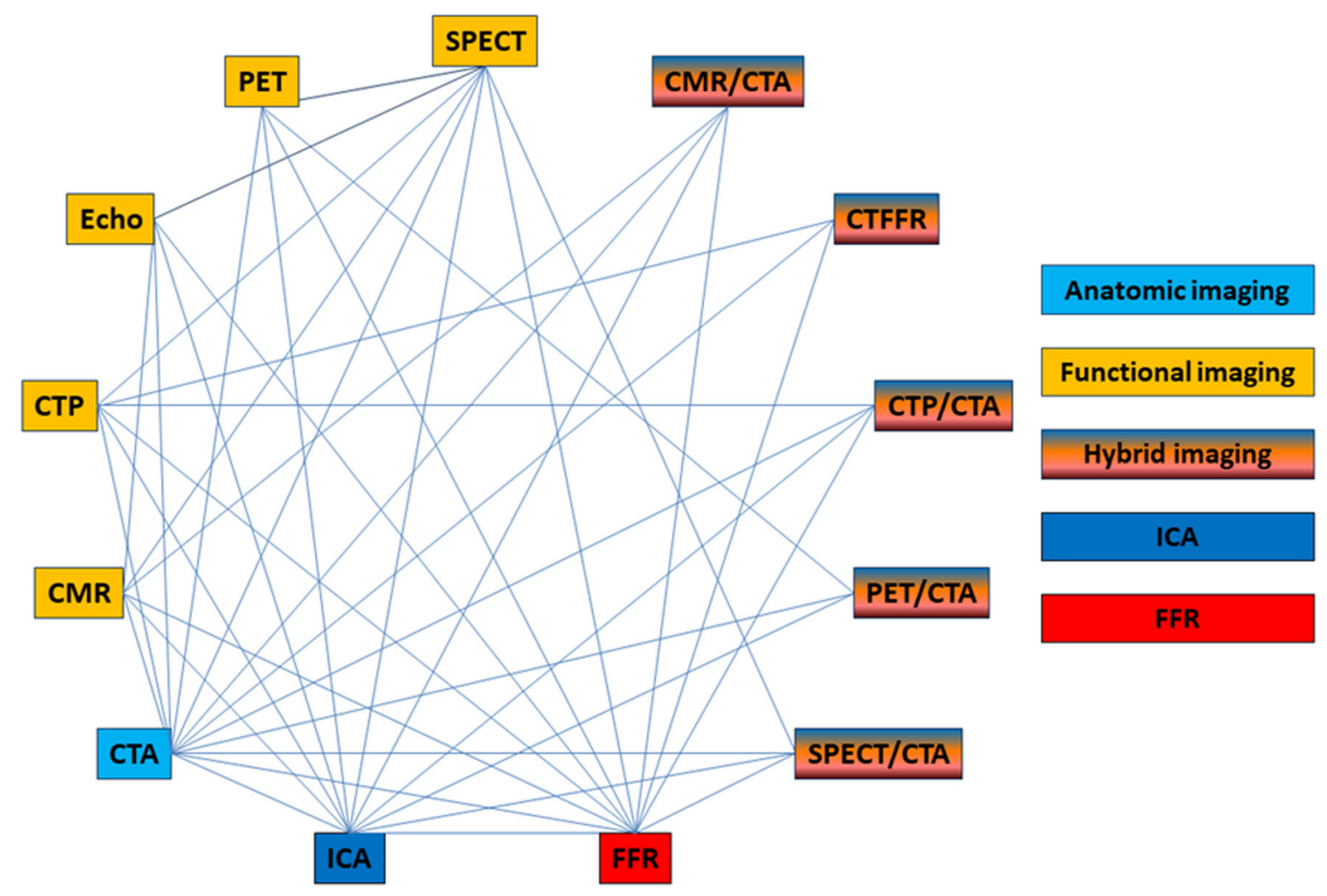

Figure 1. Evidence geometry distinguishing anatomic, functional, and hybrid imaging tests. $C M R$, cardiac magnetic resonance; $C T A$, computed tomography angiography; $C T F F R$, computed tomography-fractional flow reserve; $C T P$, computed tomography perfusion; Echo, stress echocardiography; $F F R$, fractional flow reserve; ICA, invasive coronary angiography; PET, positron emission tomography; SPECT, single photon emission computed tomography.

tests for small study effects/publication bias (Table 1S) (Figures $8 \mathrm{~S}$ to $13 \mathrm{~S}$ ). Specifically, anatomic imaging proved best for sensitivity $(0.94$ [0.90-0.96]) but performed poorly for specificity $(0.46[0.38-0.55])$, with similar ancillary findings, such as $+\mathrm{LR}=1.7(1.5-2.1)$, $-\mathrm{LR}=0.14(0.09-0.22)$, DOR $=12.2(7.0-21.4)$, and AUC $=0.93$ (0.88-0.98). Hybrid imaging provided the best overall performance, with sensitivity $=0.87(0.83$ $0.90)$, specificity $=0.82(0.76-0.87),+\mathrm{LR}=4.9(3.6-$ $6.9),-\mathrm{LR}=31.0(19.6-49.3), \mathrm{DOR}=31.0(19.6-49.3)$, and $\mathrm{AUC}=0.94(0.92-0.96)$.

\section{Multivariate Meta-analysis}

Multivariate meta-analysis was also characterized by significant statistical heterogeneity and inconsistency when focusing on sensitivity, specificity and + LR (all $P<0.001, I^{2} \geq 90 \%$ ), whereas when focusing on - LR no significant heterogeneity or inconsistency was found $\left(P=1, I^{2}=1 \%\right)$ (Tables 5, 2S). Inferential analysis showed that anatomic imaging was most accurate in comparison to ICA (sensitivity $=0.96$ [0.95-0.97], specificity $=0.83[0.81-0.85],+\mathrm{LR}=12.3$ [8.7-15.9], and $-\mathrm{LR}=0.16[0.05-0.27]$ ), with hybrid imaging markedly improving results of functional imaging in terms of sensitivity (from 0.84 [0.83-0.85] to 0.91[0.89$0.93]$ ), specificity (from 0.77 [0.76-0.78] to 0.87 [0.840.90]), + LR (from 6.1 [5.4-6.7] to 13.6 [8.3-19.0]), and - LR (from 0.32 [0.29-0.35] to 0.17 [0-0.39]). Conversely, hybrid imaging was associated with the best overall diagnostic performance (sensitivity $=0.88$ [0.86-0.91], specificity $=0.82[0.77-0.87],+\mathrm{LR}=7.2$ $[0-15.4], \quad-\mathrm{LR}=0.27 \quad[0.12-0.42])$, with anatomic imaging displaying the best sensitivity (0.95 [0.93$0.97])$ yet at the price of very poor specificity $(0.46$ [0.38-0.55]), with consistent results for + LR (2.0 [1.6$2.5])$ and - LR (0.38 [0.16-0.61]).

\section{Additional Analyses}

Subgroup pairwise analyses using ICA as reference test showed largely similar results to the main analyses. Anatomic imaging proved as the most accurate test (sensitivity $=0.95[0.94-0.96]$, specificity $=0.83[0.81$ - 
$0.85],+\mathrm{LR}=5.7$ [5.0-6.5], $-\mathrm{LR}=0.06$ [0.05-0.07], DOR $=95.6 \quad[73.2-122.7], \quad$ AUC $=0.98 \quad[0.96-0.99])$ (Table 3S). Instead, when using FFR as reference test, the best diagnostic performance was provided by CMR, CTP, and CTP/CTA. Yet, both sets of pairwise analyses were limited in scope given the exclusion of the PET, CMR/CTA, CTFFR, PET/CTA and SPECT/CTA arms for paucity of data (Table 4S), and still fraught with significant statistical heterogeneity and inconsistency (Tables 5S and 6S). Finally, small study effects appeared significant for the SPECT vs. ICA and the CTP vs ICA comparisons (Table 5S).

Exploratory multivariate meta-regression showed that year of publication, sample size, and prevalence of CAD were all significantly impactful on the results of the analyses. In particular, year of publication had a complex association with diagnostic yield (Table 7S). Briefly, more recent studies provided similar or less optimistic estimates of diagnostic accuracy for most comparisons, with the notable exception of sensitivity of anatomic imaging and + LR of functional imaging. Larger samples were associated in general with worse diagnostic yield, excluding sensitivity and + LR of anatomic imaging. Finally, higher prevalence of CAD was associated with similar or worse diagnostic performance, with the exception of sensitivity of functional imaging and hybrid imaging.

\section{DISCUSSION}

This umbrella review and multivariate meta-analysis provides the most recent and comprehensive synthesis of the evidence base on non-invasive imaging tests for the diagnosis of CAD. It is based on the inclusion of several recent and comprehensive reviews, their appraisal and summary, and finally the re-analysis by means of pairwise and multivariate methods of all study-level findings, focusing on the comparison between anatomic imaging tests (i.e., CTA), functional imaging tests, and hybrid imaging tests. Its findings are that CTA offers satisfying diagnostic accuracy for angiographically significant $\mathrm{CAD}$, based primarily on the very high per-patient sensitivity. Conversely, hybrid imaging appears as the best diagnostic test for functionally significant CAD, given the evidently limited specificity of CTA in such setting.

CAD is the most common and most important cause of ischemic heart disease, with exceedingly important impactful implications on morbidity and mortality world-wide. ${ }^{2,3}$ Three types of non-invasive imaging diagnostic tests for CAD are available: anatomic imaging, functional imaging, and hybrid imaging, which combines the former two tests. ${ }^{4,5,42,43}$ Several systematic reviews have been published synthesizing 
Table 5. Results of multivariate meta-analysis

\begin{tabular}{clcccl}
\hline Reference test & \multicolumn{1}{c}{ Index test } & Sensitivity & Specificity & $+\mathbf{L R}$ & \multicolumn{1}{c}{$\mathbf{L R}$} \\
\hline Invasive coronary & Anatomic imaging & $0.96(0.95-0.97)$ & $0.83(0.81-0.85)$ & $12.3(8.7-15.9)$ & $0.16(0.05-0.27)$ \\
angiography & Functional imaging & $0.84(0.83-0.85)$ & $0.77(0.76-0.78)$ & $6.1(5.4-6.7)$ & $0.32(0.29-0.35)$ \\
& Hybrid imaging & $0.91(0.89-0.93)$ & $0.87(0.84-0.90)$ & $13.6(8.3-19.0)$ & $0.17(0-0.39)$ \\
Invasive fractional & Anatomic imaging & $0.95(0.93-0.97)$ & $0.46(0.38-0.55)$ & $2.0(1.6-2.5)$ & $0.38(0.16-0.61)$ \\
flow reserve & Functional imaging & $0.79(0.75-0.83)$ & $0.81(0.77-0.85)$ & $6.3(4.5-8.2)$ & $0.42(0.33-0.50)$ \\
& Hybrid imaging & $0.88(0.86-0.91)$ & $0.82(0.77-0.87)$ & $7.2(0-15.4)$ & $0.27(0.12-0.42)$
\end{tabular}

$+L R$, positive likelihood ratio; $-L R$, negative likelihood ratio

diagnostic test accuracy studies for coronary artery disease focusing on the same index and reference tests. ${ }^{14,21,41}$ Their results already provide support for the use of hybrid imaging as the best strategy to maximize sensitivity while maintaining adequate specificity. However, all published reviews (and even more so individual diagnostic test accuracy studies) were limited by a narrow scope and focus on few competing tests.

Umbrella reviews represent a novel and comprehensive means to summarize extensive evidence on a clinical issue by collecting different systematic reviews published at large on a given topic, and when combined with potent statistical methods such as pairwise and multivariate meta-analysis, may provide a poignant and inferential synthesis of salient effect estimates. ${ }^{15-17}$ Indeed, we performed the most comprehensive overview of reviews of diagnostic test accuracy on non-invasive imaging for CAD to date. We identified several highquality systematic reviews, which highlighted the excellent sensitivity and favorable specificity of CTA for angiographically significant CAD. Most recent reviews also highlighted the beneficial diagnostic accuracy profile of functional tests for functionally significant CAD. Most importantly, adding CTA to functional imaging tests in a logic of hybrid imaging further improved the overall diagnostic accuracy for functionally significant $\mathrm{CAD}$, mainly by increasing the specificity of CTA, which was quite unsatisfactory (averaging 50\%).

These were the main findings, which were largely confirmed at exploratory subgroup analysis focusing on individual tests. CTA proved as the best test for anatomically significant $\mathrm{CAD}$, whereas functional and anatomical-functional (i.e., hybrid) tests proved best for functionally significant CAD. Further studies are however required to better compare the individual diagnostic accuracy of different functional and hybrid tests. Thus, in the meanwhile individualized decision-making should consider, on top of scholarly evidence, expertise, availability, cost, radiation exposure and prognostic detail, among the many important factors.
Indeed, on top of diagnostic accuracy features focusing on per-patient diagnosis of CAD, other important aspects of non-invasive imaging tests should be considered, such as ancillary findings (e.g., calcium score at computed tomography, valve assessment at echocardiography, viability assessment at SPECT, among the many others). In addition, the validated prognostic role of a given test, especially considering the possible decision to proceed with coronary revascularization or medical management only, should not be disregarded. In such sense, the largest body of evidence supports the role of CMR and SPECT. Nonetheless, the logic of current technological advancements and evidence synthesis exercises such as the present one is to proceed smoothly toward the integration of different tests in a holistic logic which builds upon each test strengths and overcomes the corresponding limitations in an integrative fashion.

Despite our careful efforts, this work has several limitations. First, being a very comprehensive umbrella review with subsequent meta-analysis we did not proceed with actual data extraction from individual diagnostic test accuracy studies but relied instead on data already abstracted in the shortlisted reviews. Second, we focused only on per-patient diagnosis of $\mathrm{CAD}$, appraised in a dichotomous fashion, and thus disregarding the continuum of coronary disease in terms of severity, extent, and involvement of proximal/dominant vessels. In addition, we did not focus on therapeutic choice or prognosis, and thus the present review cannot provide formal guidance on these two very important aspects. Moreover, we did not collect data on radiation exposure or adverse events following inaccurate diagnosis, which are both very important features for decision-making. ${ }^{44}$ In addition, most analyses were fraught with significant heterogeneity and inconsistency, thus casting a light of approximation and limited internal validity on pooled effect estimates. Indeed, clinical heterogeneity and statistical inconsistency may affect the precision and applicability of summary effect estimates from our umbrella review. 
However, the purpose of any umbrella review (i.e., overview of systematic reviews) is precisely to explicitly appraise the totality of evidence on a given clinical topic. $^{15}$ Accordingly, by purposefully pooling data from so many studies on so many different comparative imaging tests, we could highlight substantial variability in effect among different imaging modalities, but still a substantial benefit of combining anatomic and functional tests, irrespective of their core components, to increase simultaneously sensitivity and specificity. In addition, subgroup and meta-regression analyses highlight important differences between components of functional imaging tests, and also the importance of year of publication, sample size, and prevalence of $\mathrm{CAD}$ in driving diagnostic accuracy measures. Finally, as for any umbrella review focusing on a well researched topic, overall findings cannot be overly surprising or unexpected. Accordingly, the fact that anatomic imaging compares well with ICA and functional imaging with FFR could be inferred by many prior works. However, none had the sample or comprehensiveness of our work. In addition, the fact that several different functional imaging tests can similarly benefit and complement the results of anatomic tests to boost the overall diagnostic accuracy of non-invasive diagnostic testing for CAD can only be validly inferred by a comprehensive summary of the evidence base such as our present work.

In conclusion, non-invasive hybrid imaging tests appear superior to anatomic or functional only tests to diagnose ischemia-provoking coronary lesions, whereas anatomic imaging is best to diagnose and/or rule out angiographically significant CAD. Eventually, the ultimate value of any cardiovascular test rests on its ability to predict events, change management, and improve events. Thus, dedicated prospective studies are needed to more formally test the potential benefits of noninvasive hybrid imaging tests in patients with or suspected CAD.

\section{NEW KNOWLEDGE GAINED}

Coronary artery disease is the most common and most relevant cause of ischemic heart disease, with exceedingly important implications on morbidity and mortality world-wide. Three types of non-invasive imaging diagnostic tests for CAD are available: anatomic imaging, functional imaging, and hybrid imaging, which combines the former two tests. A number of systematic reviews have been published synthesizing diagnostic test accuracy studies for CAD focusing on the same index and reference tests. Yet, there is still uncertainty on their comparative diagnostic accuracy.
Umbrella reviews represent a novel and comprehensive means to summarize extensive evidence on a clinical issue by collecting different systematic reviews published at large on a given topic, and when combined with potent statistical methods such as pairwise and multivariate meta-analysis, may provide a poignant and inferential synthesis of salient effect estimates.

Stemming from a total of 22 meta-analyses and 661 clinical studies, pairwise meta-analysis using ICA as reference test suggested the superior accuracy of anatomic imaging, whereas using FFR highlighted the superiority of hybrid imaging. These results were confirmed in direction and magnitude of effect at multivariate meta-analysis. Accordingly, hybrid imaging appears as the first-choice test for the diagnosis of ischemia-provoking coronary lesions, whereas anatomic imaging is best to diagnose or rule out angiographically significant lesions.

\section{Disclosure}

Prof. Biondi-Zoccai has consulted for Abbott Vascular and Bayer. Prof. Versaci, Prof. Iskandrian, Prof. Schillaci, Dr. A. Nudi, Prof. Frati and Dr. F. Nudi have nothing to disclose.

\section{Authors Contributions}

$G Z B$ and FN designed the study, interpreted the results and drafted the manuscript. GBZ, AN, and GF performed searches, extracted data, and appraised them. FV and AEI provided critical input on study design, data analysis, and interpretation. All authors finally approved the manuscript before submission. FN acts as guarantor.

\section{Data Sharing Statement}

All data used to generate the analyses reported in this work are attached as an online supplement.

\section{References}

1. Benjamin EJ, Blaha MJ, Chiuve SE, Cushman M, Das SR, Deo R, de Ferranti SD, Floyd J, Fornage M, Gillespie C, Isasi CR, Jiménez MC, Jordan LC, Judd SE, Lackland D, Lichtman JH, Lisabeth L, Liu S, Longenecker CT, Mackey RH, Matsushita K, Mozaffarian D, Mussolino ME, Nasir K, Neumar RW, Palaniappan L, Pandey DK, Thiagarajan RR, Reeves MJ, Ritchey M, Rodriguez CJ, Roth GA, Rosamond WD, Sasson C, Towfighi A, Tsao CW, Turner MB, Virani SS, Voeks JH, Willey JZ, Wilkins JT, Wu JH, Alger HM, Wong SS, Muntner P, American Heart Association Statistics Committee and Stroke Statistics Subcommittee. Heart Disease and Stroke Statistics-2017 Update: A Report from the American Heart Association. Circulation 2017;135:e146-603.

2. GBD 2016 Causes of Death Collaborators. Global, regional, and national age-sex specific mortality for 264 causes of death, 1980- 
2016: A systematic analysis for the Global Burden of Disease Study 2016. Lancet 2017;390:1151-210.

3. GBD 2016 Disease and Injury Incidence and Prevalence Collaborators. Global, regional, and national incidence, prevalence, and years lived with disability for 328 diseases and injuries for 195 countries, 1990-2016: a systematic analysis for the Global Burden of Disease Study 2016. Lancet 2017;390:1211-59.

4. Mann DL, Zipes DP, Libby P, Bonow RO, editors. Braunwald's heart disease: A textbook of cardiovascular medicine. Philadelphia: Saunders; 2014.

5. Fuster V, Harrington RA, editors. Hurst's the heart. New York: McGraw-Hill; 2017.

6. Froelicher VF, Myers J. Manual of exercise testing. St. Louis: Mosby; 2006.

7. Picano E. Stress echocardiography. Cham: Springer International Publishing; 2016.

8. Heller GV, Hendel RC, editors. Nuclear cardiology: Practical applications. New York: McGraw-Hill; 2017.

9. Schindler TH, George RT, Lima JAC, editors. Molecular and multimodality imaging in cardiovascular disease. Cham: Springer; 2015.

10. Budoff MJ, Shinbane JS, editors. Cardiac CT imaging: Diagnosis of cardiovascular disease. Cham: Springer; 2016.

11. Bogaert J, Dymarkowski S, Taylor AM, Muthurangu V, editors. Clinical cardiac MRI. Cham: Springer; 2012.

12. Branch KR, Haley RD, Bittencourt MS, Patel AR, Hulten E, Blankstein R. Myocardial computed tomography perfusion. Cardiovasc Diagn Ther 2017;7:452-62.

13. Tesche C, Vliegenthart R, Duguay TM, De Cecco CN, Albrecht MH, De Santis D, Langenbach MC, Varga-Szemes A, Jacobs BE, Jochheim D, Baquet M, Bayer RR Nd, Litwin SE, Hoffmann E, Steinberg DH, Schoepf UJ. Coronary computed tomographic angiography-derived fractional flow reserve for therapeutic decision making. Am J Cardiol 2017;120:2121-7.

14. Rizvi A, Han D, Danad I, Ó Hartaigh B, Lee JH, Gransar H, Stuijfzand WJ, Roudsari HM, Park MW, Szymonifka J, Chang HJ, Jones EC, Knaapen P, Lin FY, Min JK, Peña JM. Diagnostic performance of hybrid cardiac imaging methods for assessment of obstructive coronary artery disease compared with stand-alone coronary computed tomography angiography: A meta-analysis. JACC Cardiovasc Imaging 2018;11:589-99.

15. Biondi-Zoccai G, editor. Umbrella reviews: Evidence synthesis with overviews of reviews and meta-epidemiologic studies. Cham: Springer; 2016.

16. Biondi-Zoccai G, editor. Diagnostic meta-analysis: A useful tool for clinical decision making. Cham: Springer; 2018.

17. Biondi-Zoccai G, editor. Network meta-analysis: Evidence synthesis with mixed treatment comparison. Hauppauge, NY: Nova Science Publishers; 2014.

18. The Cochrane Collaboration. http://cochrane.org (last accessed on August 22, 2018).

19. International Prospective Register of Systematic Reviews. https:// www.crd.york.ac.uk/prospero/ (last accessed on August 22, 2018).

20. McInnes MDF, Moher D, Thombs BD, McGrath TA, Bossuyt PM, the PRISMA-DTA Group, Clifford T, Cohen JF, Deeks JJ, Gatsonis C, Hooft L, Hunt HA, Hyde CJ, Korevaar DA, Leeflang MMG, Macaskill P, Reitsma JB, Rodin R, Rutjes AWS, Salameh JP, Stevens A, Takwoingi Y, Tonelli M, Weeks L, Whiting P, Willis BH. Preferred reporting items for a systematic review and meta-analysis of diagnostic test accuracy studies: The PRISMADTA statement. JAMA 2018;319:388-96.

21. Chen L, Wang X, Bao J, Geng C, Xia Y, Wang J. Direct comparison of cardiovascular magnetic resonance and single-photon emission computed tomography for detection of coronary artery disease: A meta-analysis. PLoS ONE 2014;9:e88402.

22. Dai N, Zhang X, Zhang Y, Hou L, Li W, Fan B, Zhang T, Xu Y. Enhanced diagnostic utility achieved by myocardial blood analysis: A meta-analysis of noninvasive cardiac imaging in the detection of functional coronary artery disease. Int $\mathbf{J}$ Cardiol 2016;221:665-73.

23. Danad I, Szymonifka J, Twisk JWR, Norgaard BL, Zarins CK, Knaapen P, Min JK. Diagnostic performance of cardiac imaging methods to diagnose ischaemia-causing coronary artery disease when directly compared with fractional flow reserve as a reference standard: A meta-analysis. Eur Heart J 2017;38:991-8.

24. de Jong MC, Genders TS, van Geuns RJ, Moelker A, Hunink MG. Diagnostic performance of stress myocardial perfusion imaging for coronary artery disease: A systematic review and meta-analysis. Eur Radiol 2012;22:1881-95.

25. Ding A, Qiu G, Lin W, Hu L, Lu G, Long X, Hong X, Chen Y, Luo X, Tang Q, Deng D. Diagnostic performance of noninvasive fractional flow reserve derived from coronary computed tomography angiography in ischemia-causing coronary stenosis: A metaanalysis. Jpn J Radiol 2016;34:795-808.

26. Dolor RJ, Patel MR, Melloni C, Chatterjee R, McBroom AJ, Musty MD, Wing L, Coeytaux RR, Ross AK, Bastian LA, Anderson M, Kosinski AS, Sanders GD. Noninvasive Technologies for the diagnosis of coronary artery disease in women. Rockville (MD): Agency for Healthcare Research and Quality (US); 2012.

27. Gonzalez JA, Lipinski MJ, Flors L, Shaw PW, Kramer CM, Salerno M. Meta-analysis of diagnostic performance of coronary computed tomography angiography, computed tomography perfusion, and computed tomography-fractional flow reserve in functional myocardial ischemia assessment versus invasive fractional flow reserve. Am J Cardiol 2015;116:1469-78.

28. Huang JY, Huang CK, Yen RF, Wu HY, Tu YK, Cheng MF, Lu CC, Tzen KY, Chien KL, Wu YW. Diagnostic performance of attenuation-corrected myocardial perfusion imaging for coronary artery disease: A systematic review and meta-analysis. J Nucl Med 2016;57:1893-8.

29. Kiaos A, Tziatzios I, Hadjimiltiades S, Karvounis C, Karamitsos TD. Diagnostic performance of stress perfusion cardiac magnetic resonance for the detection of coronary artery disease: A systematic review and meta-analysis. Int J Cardiol 2018;252:229-33.

30. Jaarsma C, Leiner T, Bekkers SC, Crijns HJ, Wildberger JE, Nagel E, Nelemans PJ, Schalla S. Diagnostic performance of noninvasive myocardial perfusion imaging using single-photon emission computed tomography, cardiac magnetic resonance, and positron emission tomography imaging for the detection of obstructive coronary artery disease: A meta-analysis. J Am Coll Cardiol 2012;59:1719-28.

31. Li M, Du XM, Jin ZT, Peng ZH, Ding J, Li L. The diagnostic performance of coronary artery angiography with 64-MSCT and post 64-MSCT: Systematic review and meta-analysis. PLoS ONE 2014;9:e84937.

32. Goodacre S, Thokala P, Carroll C, Stevens JW, Leaviss J, Al Khalaf M, Collinson P, Morris F, Evans P, Wang J. Systematic review, meta-analysis and economic modelling of diagnostic strategies for suspected acute coronary syndrome. Health Technol Assess 2013;17:1-188.

33. Health Quality Ontario. Non-invasive cardiac imaging technologies for the diagnosis of coronary artery disease: A summary of evidence-based analyses. Ont Health Technol Assess Ser 2010;10:1-40. 
34. Chest Pain of Recent Onset: Assessment and Diagnosis. National Institute for Health and Care Excellence. https://www.nice.org. uk/guidance/cg95 (last accessed on August 22, 2018).

35. Nielsen LH, Ortner N, Nørgaard BL, Achenbach S, Leipsic J, Abdulla J. The diagnostic accuracy and outcomes after coronary computed tomography angiography vs. conventional functional testing in patients with stable angina pectoris: A systematic review and meta-analysis. Eur Heart J Cardiovasc Imaging 2014;15:96171.

36. Nudi F, Iskandrian AE, Schillaci O, Peruzzi M, Frati G, BiondiZoccai G. Diagnostic accuracy of myocardial perfusion imaging with CZT technology: systemic review and meta-analysis of comparison with invasive coronary angiography. JACC Cardiovasc Imaging 2017;10:787-94.

37. Parker MW, Iskandar A, Limone B, Perugini A, Kim H, Jones C, Calamari B, Coleman CI, Heller GV. Diagnostic accuracy of cardiac positron emission tomography versus single photon emission computed tomography for coronary artery disease: A bivariate meta-analysis. Circ Cardiovasc Imaging 2012;5:700-7.

38. Sethi P, Panchal HB, Veeranki SP, Ur Rahman Z, Mamudu H, Paul TK. Diagnostic value of noninvasive computed tomography perfusion imaging and coronary computed tomography angiography for assessing hemodynamically significant native coronary artery lesions. Am J Med Sci 2017;354:291-8.

39. Sørgaard MH, Kofoed KF, Linde JJ, George RT, Rochitte CE, Feuchtner G, Lima JA, Abdulla J. Diagnostic accuracy of static CT perfusion for the detection of myocardial ischemia. A systematic review and meta-analysis. J Cardiovasc Comput Tomogr 2016;10:450-7

40. Tan XW, Zheng Q, Shi L, Gao F, Allen JC Jr, Coenen A, Baumann S, Schoepf UJ, Kassab GS, Lim ST, Wong ASL, Tan JWC, Yeo KK, Chin CT, Ho KW, Tan SY, Chua TSJ, Chan ESY, Tan RS, Zhong L. Combined diagnostic performance of coronary computed tomography angiography and computed tomography derived fractional flow reserve for the evaluation of myocardial ischemia: A meta-analysis. Int J Cardiol 2017;236:100-6.

41. Zhou T, Yang LF, Zhai JL, Li J, Wang QM, Zhang RJ, Wang S, Peng ZH, Li M, Sun G. SPECT myocardial perfusion versus fractional flow reserve for evaluation of functional ischemia: a meta analysis. Eur J Radiol 2014;83:951-6.

42. Garrone P, Biondi-Zoccai G, Salvetti I, Sina N, Sheiban I, Stella PR, Agostoni P. Quantitative coronary angiography in the current era: principles and applications. J Interv Cardiol 2009;22:527-36.

43. Pijls NH, Tanaka N, Fearon WF. Functional assessment of coronary stenoses: can we live without it? Eur Heart J 2013;34:133544.

44. Archbold RA. Comparison between National Institute for Health and Care Excellence (NICE) and European Society of Cardiology (ESC) guidelines for the diagnosis and management of stable angina: Implications for clinical practice. Open Heart 2016;3:e00406. 